\title{
Nonlinear stay cable - bridge deck interaction
}

\author{
Benedikt Weber ${ }^{1}$ \\ ${ }^{1}$ Senior Researcher, Empa, Swiss Federal Laboratories for Materials Science and Technology, 8600 Dübendorf, Switzerland.
}

\begin{abstract}
Nonlinear coupling between stay cables and bridge deck can cause internal resonance. This coupling is due to the length change of the cables when vibrating. During one cycle of transverse motion, the cable length is reduced twice, once for the positive and once for negative displacement. This internal resonance occurs when the natural frequency of the deck is twice the natural frequency of the cable. It is thus also called 2:1 resonance.
\end{abstract}

Tests have been performed at Empa on a full-size model bridge in the laboratory. The cable frequency has been tuned to half of the deck frequency by attaching additional masses. The bridge was excited by an electromechanical shaker with a frequency near the resonance frequency of the deck.

Some interesting phenomena can be observed: 1) Internal resonance only occurs if the natural frequency of the cables is about half the natural frequency of the deck. 2) Internal resonance only occurs for excitation frequencies near the resonance frequency of the deck. Outside a certain frequency range, the cables mainly act as flexible supports for the deck. 3) The transition from one regime to the other takes place at different frequencies for an ascending or a descending frequency sweep. 4) Internal resonance only occurs above a critical level of excitation. 5) Increasing the excitation force above the critical value mainly increases the displacement of the cables while the deck movement is hardly affected (saturation phenomenon).

To better understand this unexpected behavior, the system is modelled as a two-degree-of-freedom system including nonlinear coupling. For the steady-state case, this nonlinear system is then solved by perturbation methods. Although the mathematics are a bit involved, the analytical solutions are very helpful for understanding and interpreting the experimental results, which otherwise appear strange sometimes.

Keywords: Internal resonance, nonlinear dynamics, perturbation methods, cable-stayed bridges, saturation phenomenon.

\section{INTRODUCTION}

Most civil engineering structures behave essentially linear. Even if they exhibit some nonlinearities, they can usually still be analyzed with linear models but with amplitude-dependent parameters. It is typically observed that the damping increases with displacement amplitudes while natural frequencies decrease. When analyzing such structures, the superposition principle applies and when excited with a harmonic force, all quantities respond with that very same frequency. Modes with higher frequencies are only excited by higher frequency components of the exciting force. This linear behavior is the basis of all modal analysis and system identification methods.

The nonlinearities we present in this paper are completely different. Geometric nonlinearities can introduce coupling between modes having natural frequencies with a ratio of 2:1. Although such nonlinearities are still considered weak, they can lead to quite unexpected behavior. An example of internal coupling in a cable-stayed steel bridge has been reported in [1].

The internal resonance can be explained intuitively by the idealized example sketched in Figure 1. The model consists of a mass on a spring to which a vertical cable is connected. When the cable is displaced to either side, it pulls up the mass, whereas in the neutral position, the force on the mass is released. Thus, one cycle of cable motion corresponds to two cycles of the mass movement.

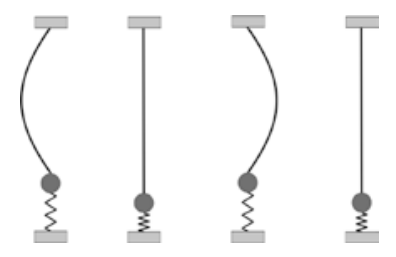

Figure 1. Sketch for visualizing the 2:1 internal resonance. 
Cable vibrations in bridges have been investigated in the literature to some extent. Some investigations only considered onedegree-freedom systems of a cable with a prescribed support motion but including sag [2-3]. These systems typically show an instability region in the frequency-amplitude diagram where infinite displacements occur. Two-degree-of-freedom systems with out-of-plane motion have been studied in [4-5]. In contrast to the in-plane motion, the out-of-plane motion of an inclined cable has no linear coupling with the bridge deck but the principal behavior is similar to the one considered here. Theoretical results on different kinds of nonlinear interactions are presented in [6]. Besides the internal resonance considered in this paper, the reference also treats nonlinear coupling due to angle variation.

References [3-5] also include experimental results, but only on small-scale laboratory systems. Tests on real bridges are rare. In [7], a large cable-stayed bridge was analyzed experimentally and cables with natural frequencies of half the deck frequency were identified. However, the authors conclude that the amplitudes were too low for internal resonance. First results of the experiments described in this paper have been presented previously [8].

\section{EXPERIMENTAL RESULTS}

Experimental investigations have been performed on a cable-stayed laboratory bridge at Empa, shown in Figure 2. The bridge is $20 \mathrm{~m}$ long and $2.5 \mathrm{~m}$ wide with a pylon of $7.5 \mathrm{~m}$ height. The deck is made of GFRP-profiles. A pair of cables, $9.25 \mathrm{~m}$ long, connects the deck to the pylon. To adjust the cable frequency to half of the deck frequency, masses were added to the cables every $0.4 \mathrm{~m}$. To force the cables to vibrate in their vertical plain, guides have been installed as shown in Figure $2 b)$. An excitation force was applied to the deck by an electromechanical shaker. The shaker was placed near the cable support. By slightly changing its position, it was possible to fine-tune the deck frequency. The bridge deck was excited harmonically varying the frequency or the amplitude of the force in several steps. For each step, a sample of 60 seconds was registered. This period was sufficient to reach steady-state conditions. Accelerations were measured on the deck at both cable supports and on both cables at mid-length. The shaker force has been calculated from the accelerations of the shaker and the acceleration of the moving mass. Other quantities have been measured but are not reported here.

As described above, internal resonance can occur when the cable frequency is close to half the deck frequency. When applying a harmonic force with a frequency close to the deck frequency, the deck vibrates with the excitation frequency, whereas, due to the nonlinear coupling, the cable vibrates with half the excitation frequency. However, due to the inclination of the cable, there is also a linear coupling exciting the cable directly with motion of the deck, and the deck with the motion of the cable. To make the analysis more clear, the responses due to the linear coupling are filtered out in all plots. Thus, for the deck, always the harmonic corresponding to the excitation frequency (denoted as mode 1) is shown, and for the cables, the harmonic corresponding to half of the excitation frequency (denoted as mode 1/2) is plotted.

The amplitudes of the deck and the cables are plotted in Figure 3. For the deck (Figure 3a), the amplitudes at the centerline are plotted, calculated from the mean acceleration between the two cable supports. For the cables, one needs to be careful with the definition of the amplitudes. Since the cables vibrate sometimes in phase, sometimes out of phase, the amplitude of the mean is zero for the out-of-phase motion, whereas the mean of the amplitudes always gives a positive value without considering the phase between the two cables. Note that the out-of-phase motion of the cables is coupled with torsion of the deck, but with the frequency of the cable, not with the frequency of the deck. In Figure 3b), we show the mean of the two amplitudes. For ascending frequencies, internal resonance starts occurring at $3.85 \mathrm{~Hz}$ and ends at $4.2 \mathrm{~Hz}$. Outside this frequency range, the cables have no harmonic contribution with half the excitation frequency. The deck amplitude shows two valleys in this range. For descending frequencies, the range of internal resonance extends down to $3.7 \mathrm{~Hz}$. Observing this difference between ascending and descending excitation frequencies for the first time can be quite confusing.

a)

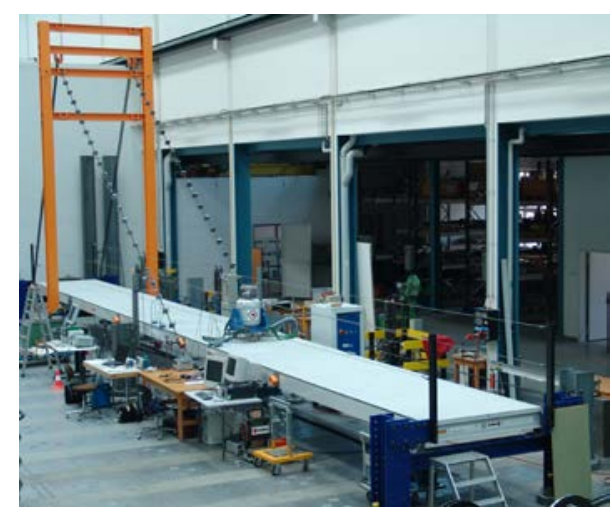

b)

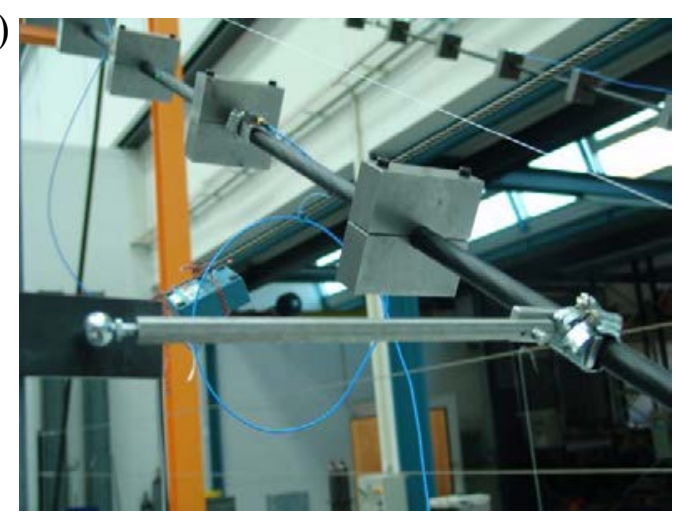

Figure 2. a) Laboratory bridge, b) cable with added mass and guide. 
To distinguish the in-phase and the out-of-phase motion of the cables, we can also look at the mean and half the difference of the two cable accelerations. The amplitudes of these values are plotted in Figure 4 a) and b) for ascending and descending excitation frequencies, respectively. For the in-phase motion, without torsion of the deck, the difference is zero, whereas for the out-of-phase motion, with torsion of the deck, the mean is zero. Clearly, the cross-over frequency is around $4 \mathrm{~Hz}$. Below this frequency, the cables vibrate in phase; above this frequency, they vibrate out of phase. The amplitudes, either in-phase or out-of-phase show the same picture as in Figure 3b).

a)

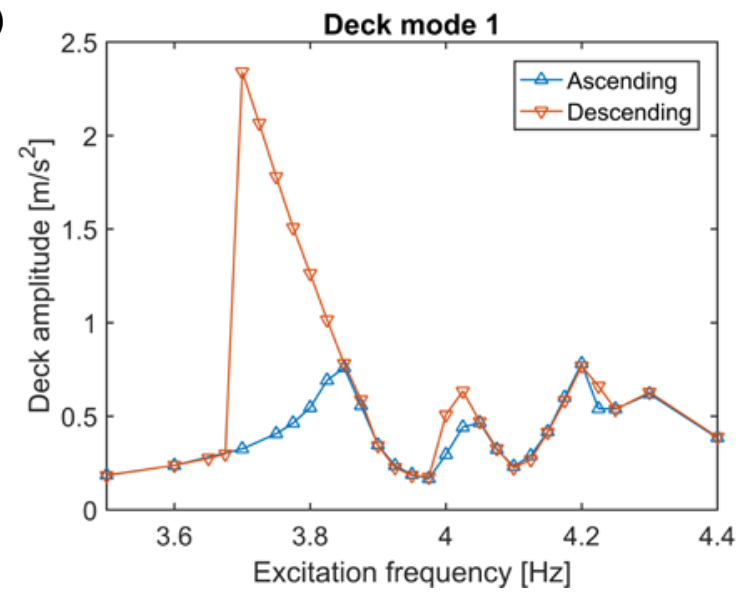

b)

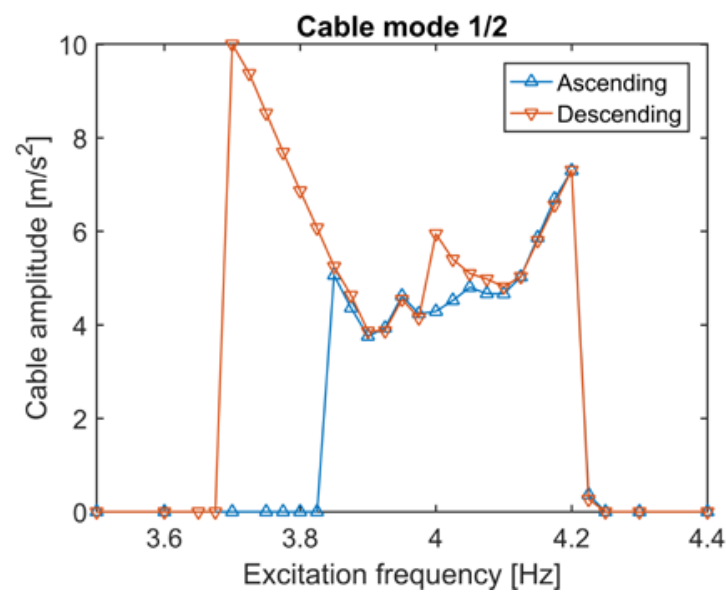

Figure 3. Frequency response of a) deck and b) cables, due to harmonic excitation with amplitude $120 \mathrm{~N}$.

a)

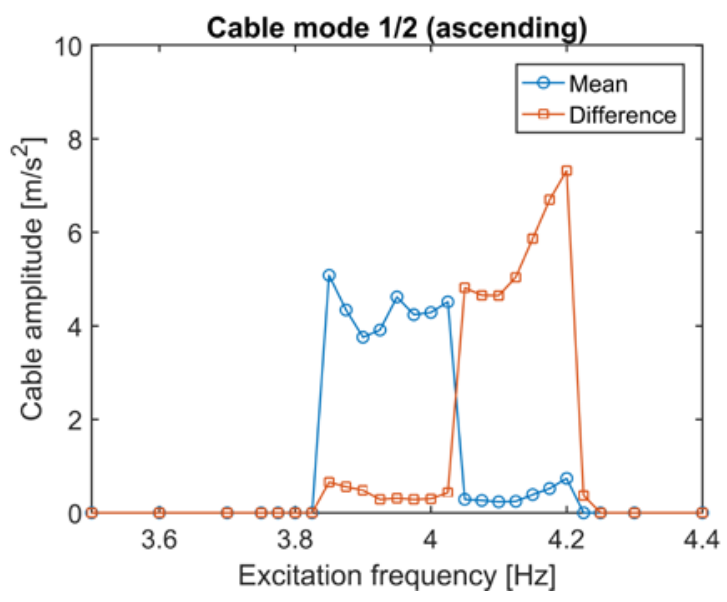

b)

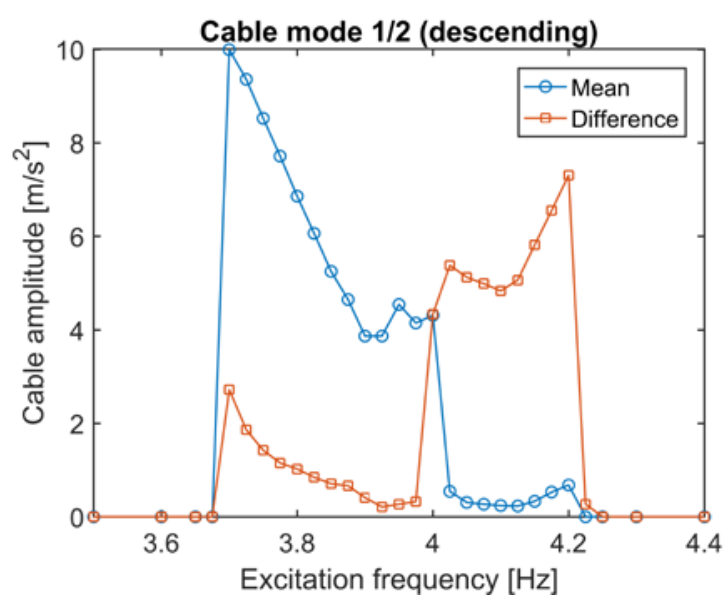

Figure 4. Frequency response of cables with a) ascending and b) descending excitation frequency.

a)

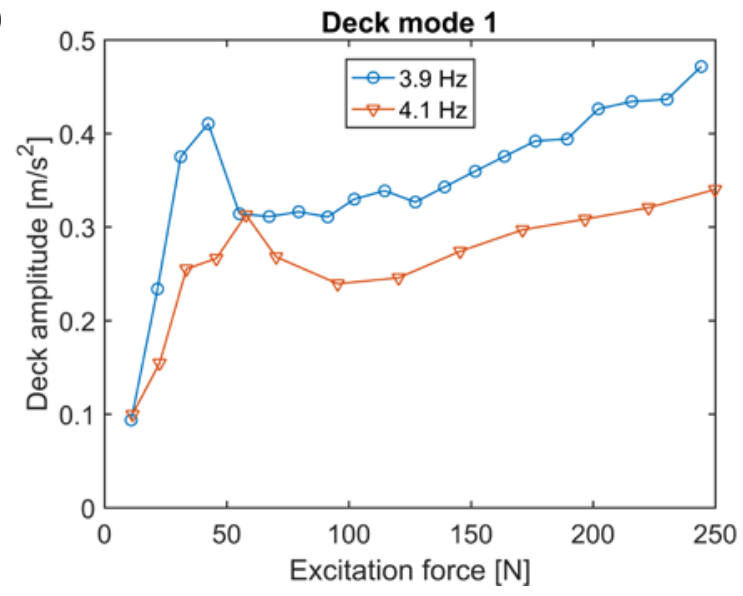

b)

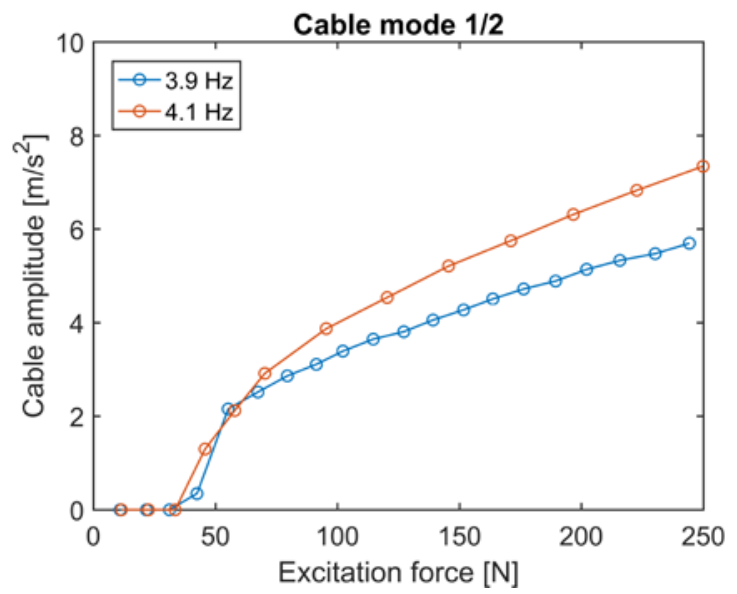

Figure 5. Amplitude response of a) deck and b) cables at two selected frequencies. 
To complete the picture, experiments were also conducted with varying the amplitude of the excitation force while keeping the frequency constant. The most interesting results are for excitation frequencies $3.9 \mathrm{~Hz}$ and $4.1 \mathrm{~Hz}$. The acceleration amplitudes of the deck and the cables are shown in Figure 5. For a low level of the excitation force, the deck amplitude increases linearly, while the cables have no harmonic contribution with a frequency of half the excitation frequency. Once the critical value of the excitation force has been reached, the deck amplitude decreases and then recovers slowly with increasing excitation force. Most of the energy goes into the cables. This is called the saturation phenomenon.

\section{THEORY}

For the formulation of the differential equations describing the combined motion of the deck and the cable, we use Lagrange's equations of motion [9], similarly as in [3-4]. The basic layout of the deck-cable system is sketched in Figure 6. The geometry is defined by the length $\ell$ of the cable and the inclination angle $\theta$. The deck is represented by the mass $M$ and the spring with stiffness $K$. The properties of the cable are the stiffness $E A$ and the distributed mass $m$. The initial tension of the cable is $N_{0}$.

The displacement of the deck is described by the variable $u$, the displacement of the cable is described by the function $y(x)$ measured from the chord, which itself is moving with the deck. Not included in this idealized model are the flexibility of the pylon and the cable sag, which modify the cable frequency [10]. These effects are considered indirectly by taking the measured eigenfrequencies in the numerical model.

The potential energy of the cable depends on the stretching of the cable, which is due to the elongation of the chord when the deck moves down and due to the lateral movement of the cable. Assuming a cable shape measured from the chord as $y=v \sin \pi x / \ell$, the cable stretching is given as

$$
\Delta \ell=u \sin \theta+\int_{0}^{\ell} \sqrt{1-y^{\prime}} d x-\ell \approx u \sin \theta+\frac{1}{2} \int_{0}^{\ell} y^{\prime} d x=u \sin \theta+\frac{v^{2} \pi^{2}}{4 \ell}
$$

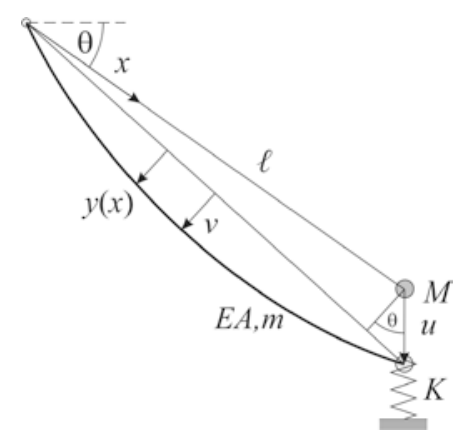

Figure 6. Layout of deck-cable system

Considering the initial cable tension $N_{0}$, the potential energy of the deck and the cable is

$$
V=N_{0} \Delta \ell+\frac{1}{2} \frac{E A}{\ell} \Delta \ell^{2}+\frac{1}{2} K_{\text {deck }} u^{2}-u N_{0} \sin \theta=N_{0} \frac{\pi^{2}}{4 \ell} v^{2}+\frac{1}{2} \frac{E A}{\ell}\left(u \sin \theta+\frac{\pi^{2}}{4 \ell} v^{2}\right)^{2}+\frac{1}{2} K_{\text {deck }} u^{2}
$$

Note that, in the potential energy, the initial cable tension gives rise to linear terms with respect to the cable elongation and the deck displacement. The kinetic energy is

$$
T=\frac{1}{2} \int_{0}^{\ell} m\left(\dot{y}(x)+\frac{x}{\ell} \dot{u} \cos \theta\right)^{2} d x+\frac{1}{2} M \dot{u}^{2}=\frac{m}{2}\left(\frac{\ell}{2} \dot{v}^{2}+2 \cos \theta \frac{\ell}{\pi} \dot{u} \dot{v}+\cos ^{2} \theta \frac{\ell}{3} \dot{u}^{2}\right)+\frac{1}{2} M \dot{u}^{2}
$$

Introducing the Lagrangian defined by $L=T-V$, the equations of motion are

$$
\frac{d}{d t}\left(\frac{\partial L}{\partial \dot{u}}\right)-\frac{\partial L}{\partial u}=Q \text { and } \frac{d}{d t}\left(\frac{\partial L}{\partial \dot{v}}\right)-\frac{\partial v}{\partial u}=0
$$

Applying Eqs. (4) to the kinetic and potential energy and neglecting third-order terms, the equation of motion becomes: 


$$
\left[\begin{array}{cc}
M+\cos ^{2} \theta \frac{m \ell}{3} & \cos \theta \frac{m \ell}{\pi} \\
\cos \theta \frac{m \ell}{\pi} & \frac{m \ell}{2}
\end{array}\right]\left[\begin{array}{l}
\ddot{u} \\
\ddot{v}
\end{array}\right]+\left[\begin{array}{cc}
K_{d e c}+\sin ^{2} \theta \frac{E A}{\ell} & 0 \\
0 & N_{0} \frac{\pi^{2}}{2 \ell}
\end{array}\right]+\left[\begin{array}{l}
u \\
v
\end{array}\right]+\sin \theta \frac{\pi^{2} E A}{4 \ell^{2}}\left[\begin{array}{c}
v^{2} \\
2 u v
\end{array}\right]=\left[\begin{array}{c}
F \cos \Omega t \\
0
\end{array}\right]
$$

Notice how the Lagrange equations automatically yield all linear and nonlinear coupling terms, which would be difficult to obtain directly. From Eq. (5) we see that the deck mass and stiffness are augmented by cable contributions and there is a mass coupling between deck and cable. More interesting are the nonlinear contributions: The term $v^{2}$ in the deck equation stems from the longitudinal cable force due to the cable displacement. The term $u v$ in the cable equation takes into account the variability of the cable tension due the movement of the deck.

The linear coupling due to the inclination of the cable can be removed by the usual transformation into modal coordinates:

$$
\left[\begin{array}{l}
u \\
v
\end{array}\right]=\left[\begin{array}{ll}
P_{11} & P_{12} \\
P_{21} & P_{22}
\end{array}\right]\left[\begin{array}{l}
q \\
r
\end{array}\right]
$$

Pre-multiplying by the transposed transformation matrix, the mass and the stiffness matrix can be uncoupled:

$$
\mathbf{P}^{T} \mathbf{M P}=\mathbf{I} \quad \text { and } \quad \mathbf{P}^{T} \mathbf{K P}=\operatorname{diag}\left(\omega_{1}, \omega_{2}\right)
$$

For the nonlinear terms, the transformation introduces all possible quadratic combinations of the variables $q$ and $r$ :

$$
\begin{aligned}
& \ddot{q}+\omega_{1}^{2} q+d_{1} q^{2}+d_{2} q r+d_{3} r^{2}=P_{11} F \cos \Omega t \\
& \ddot{r}+\omega_{2}^{2} r+d_{4} q^{2}+d_{5} q r+d_{6} r^{2}=P_{12} F \cos \Omega t
\end{aligned}
$$

For a vertical cable $(\theta=\pi / 2)$, there is no linear coupling and $u=q$ and $v=r$. It is thus convenient to refer to the first modal equation as to the deck equation and to the second equation as to the cable equation. In this case, $\omega_{1}$ is the larger frequency and $\omega_{2} \approx 1 / 2 \omega_{1}$ is the smaller frequency. The excitation frequency is near the deck frequency: $\Omega \approx \omega_{1}$.

It is shown in Nayfeh [11] that the additional quadratic terms in Eq. (8) introduced by the linear transformation do not contribute to the internal resonance and only the term with $q r$ in the first equation and the term $r^{2}$ in the second equation need to be retained. Also the excitation force in the second equation does not contribute since its frequency $\Omega$ is not close to the eigenfrequency $\omega_{2}$. Basically, the cable inclination does not introduce any important nonlinear coupling terms and we only need to discuss the case of a vertical cable. The inclination just changes the values of the parameters but not the form of the equations.

Eqs. (8) can be solved by perturbation methods [11]. For small amplitudes, the quadratic terms are small relative to the linear terms. If we also assume the exciting force to be small and add a small damping, Eqs. (8) can be written as

$$
\begin{aligned}
& \ddot{q}+\omega_{1}^{2} q+\varepsilon\left(2 \mu_{1} \dot{q}+d_{3} r^{2}\right)=\varepsilon f \cos \Omega t \\
& \ddot{r}+\omega_{2}^{2} r+\varepsilon\left(2 \mu_{2} \dot{r}+d_{5} q r\right)=0
\end{aligned}
$$

where $\varepsilon$ is an unspecified small parameter. The modal damping values are $\varepsilon \mu_{1}=\xi_{1} \omega_{1}$ and $\varepsilon \mu_{2}=\xi_{2} \omega_{2}$. The force parameter is $\varepsilon f=P_{11} F$ and the coefficients for the nonlinear terms are $\varepsilon d_{3}=\left(P_{11} P_{22}+2 P_{12} P_{21} P_{22}\right) \sin \theta \cdot \pi^{2} E A / 4 \ell^{2}$ and $\varepsilon d_{5}=2 \varepsilon d_{3}$.

The classical method for solving Eqs. (9) is the method of Multiple Scales [11]. This method is quite involved and will not be explained here. Instead we present a more intuitive method, which also shows how the nonlinear coupling introduces internal resonance. This is the method of Harmonic Balance [11] which is only applicable to periodic solutions. We are looking at the case $\omega_{1} \approx 2 \omega_{2}$ and $\Omega \approx \omega_{1}$ and thus define the detuning parameters $\rho$ and $\sigma$

$$
\omega_{1}=2 \omega_{2}+\varepsilon \rho \text { and } \Omega=\omega_{1}+\varepsilon \sigma
$$

Expressing $\omega_{1}$ and $\omega_{2}$ in Eqs. (9) in terms of the excitation frequency $\Omega$ and the detuning parameters $\rho$ and $\sigma$ yields

$$
\begin{aligned}
& \ddot{q}+\Omega^{2} q+\varepsilon\left[2 \mu_{1} \dot{q}-2 \Omega \sigma q+d_{3} r^{2}\right]=\varepsilon f \cos \Omega t \\
& \ddot{r}+\frac{1}{4} \Omega^{2} r+\varepsilon\left[2 \mu_{2} \dot{r}-\frac{1}{2} \Omega(\rho+\sigma) \mathrm{r}+d_{5} q r\right]=0
\end{aligned}
$$


These equations already show how the deck degree of freedom $q$ is vibrating with the excitation frequency $\Omega$ and the cable degree of freedom $r$ is vibrating with half the excitation frequency. We thus assume solutions of the following form

$$
\begin{aligned}
& q=a_{1} \cos \Omega t+a_{2} \sin \Omega t \\
& r=b_{1} \cos \frac{1}{2} \Omega t+b_{2} \sin \frac{1}{2} \Omega t
\end{aligned}
$$

These solutions satisfy the order $\varepsilon^{0}$ equations identically. For the order $\varepsilon^{1}$ we need to expand the quadratic terms. Multiplication of sine and cosine functions produces sine and cosine function of sums and difference of the argument. Thus, squaring sine and cosine functions with argument $1 / 2 \Omega t$ produces sine and cosine functions with argument $\Omega t$ plus constants. Likewise multiplying sine and cosine functions with arguments $1 / 2 \Omega t$ and $\Omega t$ produces sine and cosine functions with arguments $1 / 2 \Omega t$ and $3 / 2 \Omega t$. More explicitely:

$$
\begin{aligned}
& r^{2}=\frac{1}{2} b_{1}^{2}(1+\cos \Omega t)+b_{1} b_{2} \sin \Omega t+\frac{1}{2} b_{2}^{2}(1-\cos \Omega t) \\
& q r=\frac{1}{2}\left(a_{1} b_{1}-a_{2} b_{2}\right) \cos \frac{3}{2} \Omega t+\frac{1}{2}\left(a_{2} b_{1}+a_{1} b_{2}\right) \sin \frac{3}{2} \Omega t+\frac{1}{2}\left(a_{1} b_{1}+a_{2} b_{2}\right) \cos \frac{1}{2} \Omega t+\frac{1}{2}\left(a_{2} b_{1}-a_{1} b_{2}\right) \sin \frac{1}{2} \Omega t
\end{aligned}
$$

Since the cable frequency is half of the deck frequency, the cable produces a force on the deck with the deck frequency (double cable frequency) and the deck produces a force with the cable frequency (deck frequency minus cable frequency). These relations are responsible for the internal resonance. Note also that constant terms and terms with frequency $3 / 2 \Omega$ (deck frequency plus cable frequency) occur, which do not contribute to the internal resonance. Additional terms with other frequencies would also occur if we had included all quadratic combinations from Eqs. (8). It can easily be seen that these additional terms would also not contribute to internal resonance, which justifies their omission.

The method of Harmonic Balance requires that the order $\varepsilon^{1}$ terms do not excite the system to resonance. Thus the terms containing harmonics of frequency $\Omega$ in the deck equation and the terms containing harmonics of frequency $\Omega / 2$ in the cable equation have to vanish. In this way we obtain two conditions for the coefficients $a_{1}, a_{2}, b_{1}, b_{2}$ from each equation, one from sine-terms and one from cosine terms:

$$
\begin{array}{lll}
\mu_{1} a_{1}+\sigma a_{2}-\frac{d_{3}}{2 \omega_{1}} b_{1} b_{2}=0 & \mu_{1} a_{2}-\sigma a_{1}+\frac{d_{3}}{4 \omega_{1}}\left(b_{1}^{2}-b_{2}^{2}\right)=\frac{f}{2 \omega_{1}} & \text { (deck equation) } \\
\mu_{2} b_{1}+\frac{1}{2}(\rho+\sigma) b_{2}+\frac{d_{5}}{4 \omega_{2}}\left(a_{1} b_{2}-a_{2} b_{1}\right)=0 & \mu_{2} b_{2}-\frac{1}{2}(\rho+\sigma) b_{1}+\frac{d_{5}}{4 \omega_{2}}\left(a_{1} b_{1}+a_{2} b_{2}\right)=0
\end{array} \quad \text { (cable equation) }
$$

Note that in these equations, the frequency $\Omega$ has been changed back to the eigenfrequencies $\omega_{1}$ and $\omega_{2}$ using Eqs. (10).

Since we are interested in the amplitudes of the motion, the form assumed in Eq. (12) to has to be converted to the form

$$
q=A \cos (\Omega t-\alpha) \quad \text { and } \quad r=B \cos \left(\frac{1}{2} \Omega t-\beta\right)
$$

where the amplitudes are given by $A^{2}=a_{1}^{2}+a_{2}^{2}$ and $B^{2}=b_{1}^{2}+b_{2}^{2}$. Eqs. (14) have two solutions for the amplitudes [11]. One is the trivial solution corresponding to a deck motion without cable movement. This is the solution of the linear problem:

$$
A=\frac{f}{2 \omega_{1} \sqrt{\mu_{1}^{2}+\sigma^{2}}} \quad B=0
$$

The other solution is the one with internal resonance

$$
\begin{aligned}
& A=\frac{2 \omega_{2}}{d_{5}} \sqrt{4 \mu_{2}^{2}+(\rho+\sigma)^{2}} \quad B^{2}=E \pm \sqrt{\left(\frac{2 f}{d_{3}}\right)^{2}-F^{2}} \\
& \text { where } E=\frac{8 \omega_{1} \omega_{2}}{d_{3} d_{5}}\left(\sigma(\rho+\sigma)-2 \mu_{1} \mu_{2}\right) \quad \text { and } \quad F=\frac{8 \omega_{1} \omega_{2}}{d_{3} d_{5}}\left(\mu_{1}(\rho+\sigma)+2 \sigma \mu_{2}\right)
\end{aligned}
$$

It is a remarkable result that the deck amplitude in this case is independent of the applied force. Therefore, increasing the load does not increase the deck response but only the cable response. This is called the saturation phenomenon.

A more detailed solution based on the method of multiple scales is provided in [11]. This book also discusses the stability of the solutions. Stability conditions are not derived here, but the unstable solutions are given in the plots by dashed lines. 


\section{DISCUSSION}

The results of the perturbation solutions can now be used for the interpretation of the experimental results. First recall that the two cables moved symmetrically near an excitation frequency of $3.9 \mathrm{~Hz}$ but changed to an antisymmetric pattern near an excitation frequency of $4.1 \mathrm{~Hz}$. Since the model presented here includes only one cable, it cannot reproduce both of these modes. Instead, we consider two models, one with a cable frequency near $3.9 \mathrm{~Hz}$ and one with a cable frequency near $4.1 \mathrm{~Hz}$. The higher frequency is attributable to the additional torsion stiffness mobilized in this mode. The amplitudes of the deck and the cables are compared to the perturbation solutions of the two models in Figure 7. As before, the harmonic corresponding to the excitation frequency is shown for the deck and the harmonic corresponding to half of the excitation frequency is shown for the cable. This representation filters out the response due to linear coupling. The measured amplitudes can well be interpreted by the perturbation solutions. Not only can the two valleys be interpreted, but also the different behavior for a test with ascending and descending excitation frequencies can be explained.

For the analytical model, mass and stiffness properties were taken from the physical model as much as possible. However, since the results depend heavily on the correct frequencies, these properties have been adjusted to the measured response. The cable frequencies of the two models correspond directly to the valleys of the deck response. Since the deck frequency is completely hidden by the internal resonance, some additional measurements have been performed omitting internal resonance. This has been done once by applying a low level excitation force (Figure 8a) and once by restraining the cable motion by wires connecting the center of the cables with the floor (Figure 8a). The response due to a low excitation has been scaled to the normal level. The two linear frequency responses look quite similar, although there is some difference due to the different amplitudes. The deck frequency can be estimated from the main peak around $4.1 \mathrm{~Hz}$. Besides the deck mode, the response also exhibits the second cable mode, which has not been included in the analytical model. Since the cables are tuned to half the deck frequency, their second mode (with a node at the center) is close to the deck frequency. Therefore the secondary peak in these figures is close to the first valley with internal resonance (around $3.9 \mathrm{~Hz}$ ).

a)

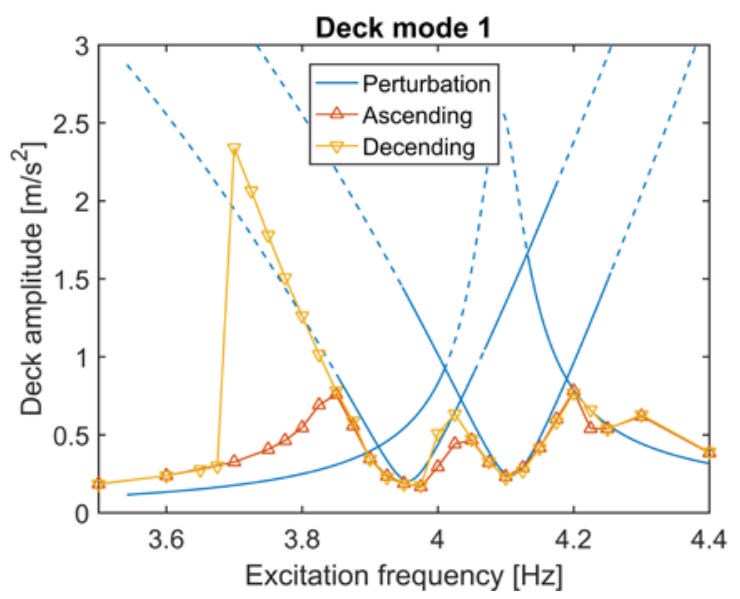

b)

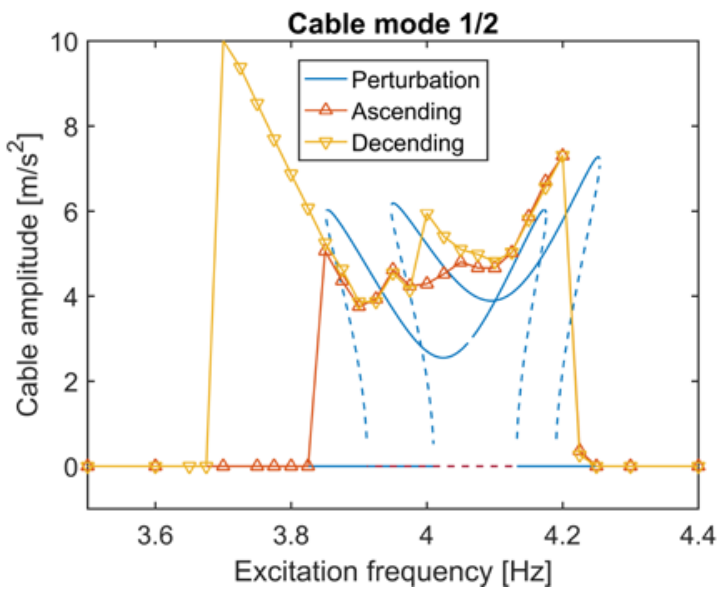

Figure 7. Frequency response of a) deck, b) cable.

a)

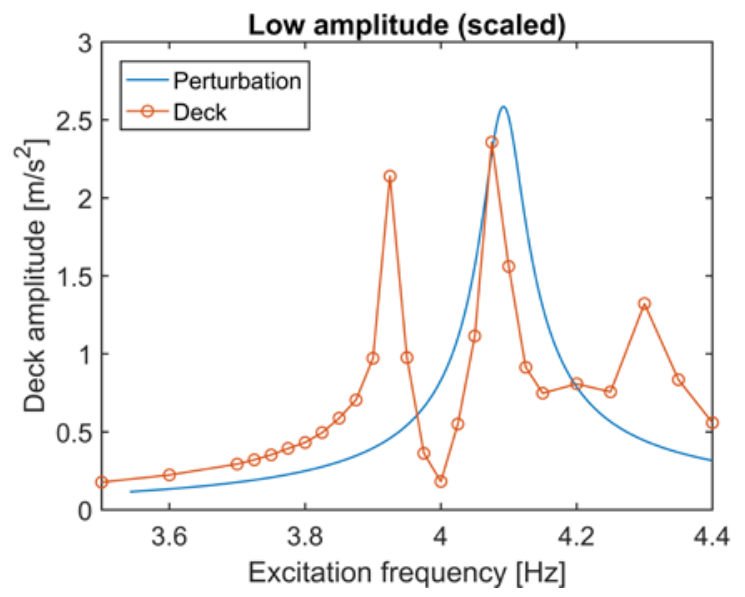

b)

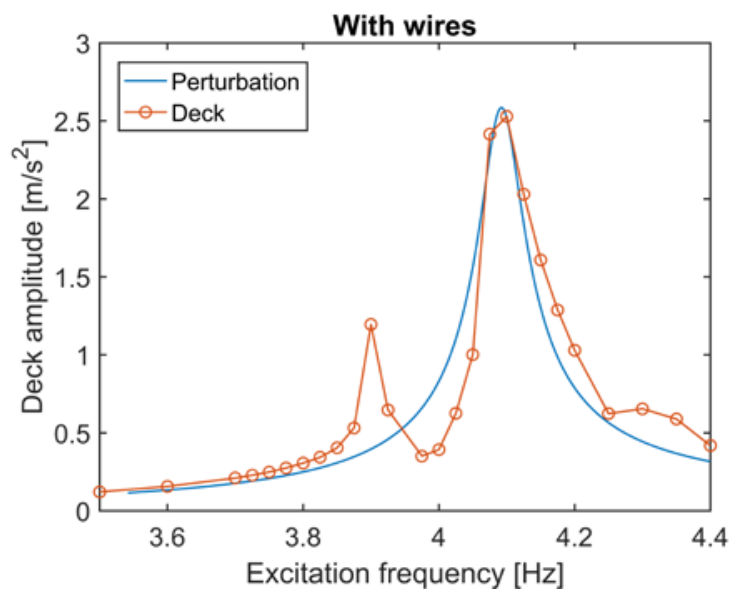

Figure 8. a) Frequency response of deck a) due to low level excitation (scaled up), b) with restrained cable motion. 
a)

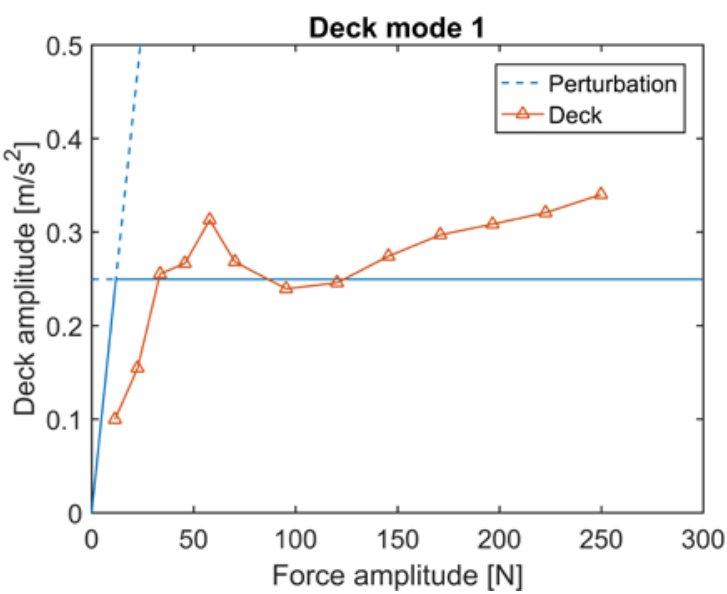

b)

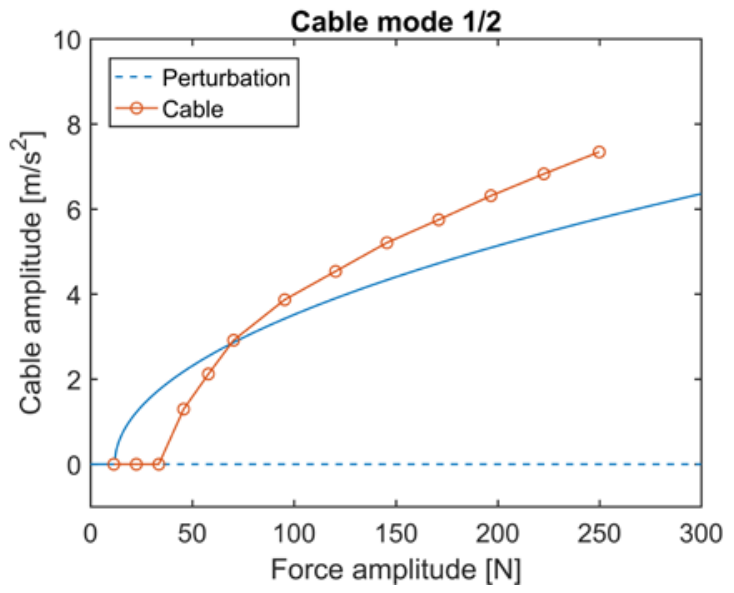

Figure 9. Response for varying excitation amplitude at $4.1 \mathrm{~Hz}$ : a) deck response, b) cable response.

The case of varying amplitude of the excitation force is shown in Figure 9. Although not all details are represented exactly by the perturbation solution, the general behavior is clearly captured. After a linear branch of the deck response with no contribution of the cable response, the deck amplitude remains more or less constant while the cable amplitude increases with increasing force amplitude.

\section{CONCLUSIONS}

Dynamic tests on a laboratory bridge have been performed to investigate the effect of internal resonance between stay cables and bridge deck. Internal resonance leads to some unusual effects which cannot be explained by the classical modal analysis. The most striking phenomenon is the coupling of two modes with different frequencies. Internal resonance only occurs when the cable frequency is tuned to half of the deck frequency. A further requirement is that the forcing function is large enough and also has a frequency close to the deck frequency.

All principal phenomena can be explained with a simple model of an inclined cable connected to a spring-mass system. The equations of motion can be derived with Lagrange's equations, which automatically yield the linear and nonlinear coupling terms. These nonlinear equations have been solved approximately by perturbation methods.

\section{REFERENCES}

[1] Hamm, P. (2007). Nicht-lineares Zusammenwirken von Brückendeck und Abspannseilen bei Fußgängerbrücken. Institut für Baustoffe und Konstruktion, Technischen Universität München.

[2] Cai, Y., and Chen, S. S. (1994). Dynamics of elastic cable under parametric and external resonances. Journal of Engineering Mechanics, 120(8), 1786-1802.

[3] Pinto da Costa, A., Martins, J. A. C., Branco, F., and Lilien, J. L. (1996). Oscillations of bridge stay cables induced by periodic motions of deck and/or towers. Journal of Engineering Mechanics, 122(7), 613-622.

[4] Perkins, N. C. (1992). Modal interactions in the non-linear response of elastic cables under parametric/external excitation. International Journal of Non-Linear Mechanics, 27(2), 233-250.

[5] Fujino, Y., Warnitchai, P., and Pacheco, B. M. (1993). An experimental and analytical study of autoparametric resonance in a 3DOF model of cable-stayed-beam. Nonlinear dynamics, 4(2), 111-138.

[6] Gattulli, V., and Lepidi, M. (2003). Nonlinear interactions in the planar dynamics of cable-stayed beam. International Journal of Solids and Structures, 40(18), 4729-4748.

[7] Caetano, E., Cunha, A., Gattulli, V., and Lepidi, M. (2008). Cable-deck dynamic interactions at the International Guadiana Bridge: On-site measurements and finite element modelling. Structural Control and Health Monitoring, 15(3), 237-264.

[8] Weber, B., and Hamm, P. (2008). Nonlinear cable-deck interaction in cable-stayed footbridges. Footbridge, Third International Conference, Porto.

[9] Meirovitch, L. (1975). Elements of vibration analysis. McGraw-Hill.

[10] Irvine, H. M. (1992). Cable structures. Dover Publications.

[11] Nayfeh, A. H. (2000). Nonlinear interactions. Wiley. 